\title{
Defining and quantifying frustration in the energy landscape: Applications to atomic and molecular clusters, biomolecules, jammed and glassy systems
}

V. K. de Souza, J. D. Stevenson, S. P. Niblett, J. D. Farrell, and D. J. Wales

Citation: The Journal of Chemical Physics 146, 124103 (2017); doi: 10.1063/1.4977794

View online: $\mathrm{http} / / / \mathrm{dx}$. doi.org/10.1063/1.4977794

View Table of Contents: http://aip.scitation.org/toc/jcp/146/12

Published by the American Institute of Physics

\section{Articles you may be interested in}

Perspective: Found in translation: Quantum chemical tools for grasping non-covalent interactions

The Journal of Chemical Physics 146, 120901120901 (2017); 10.1063/1.4978951

Microscopic derivation of particle-based coarse-grained dynamics: Exact expression for memory function The Journal of Chemical Physics 146, 124109124109 (2017); 10.1063/1.4978572

A general method for the derivation of the functional forms of the effective energy terms in coarse-grained energy functions of polymers. I. Backbone potentials of coarse-grained polypeptide chains

The Journal of Chemical Physics 146, 124106124106 (2017); 10.1063/1.4978680

Perspective: Computing (ro-)vibrational spectra of molecules with more than four atoms

The Journal of Chemical Physics 146, 120902120902 (2017); 10.1063/1.4979117

Interaction entropy for protein-protein binding

The Journal of Chemical Physics 146, 124124124124 (2017); 10.1063/1.4978893

Wavelet Monte Carlo dynamics: A new algorithm for simulating the hydrodynamics of interacting Brownian particles

The Journal of Chemical Physics 146, 124111124111 (2017); 10.1063/1.4978808

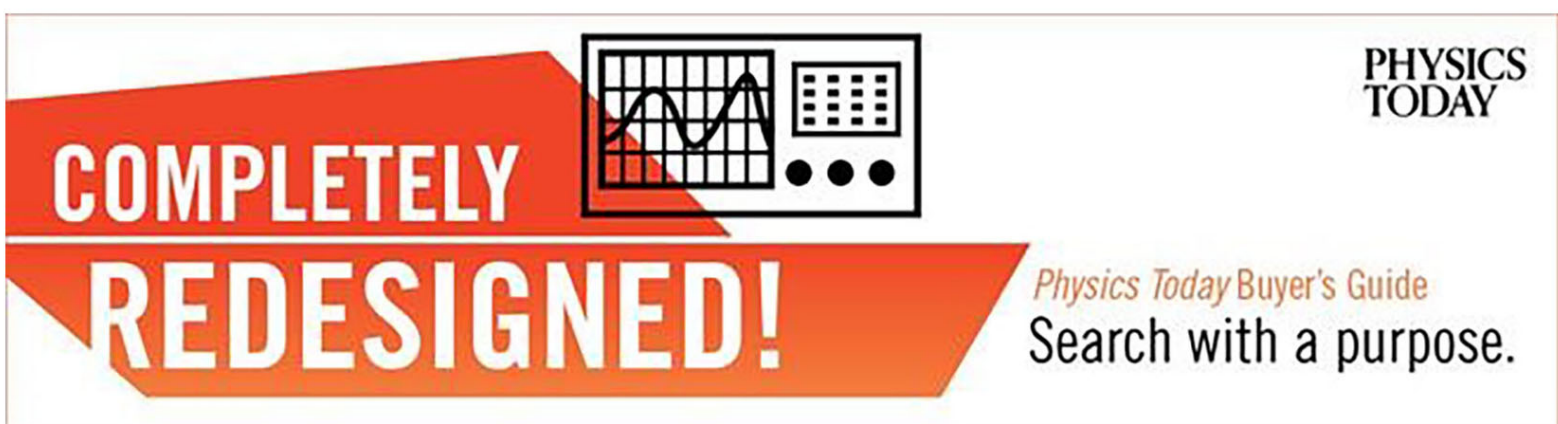




\title{
Defining and quantifying frustration in the energy landscape: Applications to atomic and molecular clusters, biomolecules, jammed and glassy systems
}

\author{
V. K. de Souza, J. D. Stevenson, S. P. Niblett, J. D. Farrell, and D. J. Wales ${ }^{a}$ ) \\ University Chemical Laboratories, Lensfield Road, Cambridge CB2 1EW, United Kingdom
}

(Received 28 October 2016; accepted 7 February 2017; published online 22 March 2017)

\begin{abstract}
The emergence of observable properties from the organisation of the underlying potential energy landscape is analysed, spanning a full range of complexity from self-organising to glassy and jammed systems. The examples include atomic and molecular clusters, a $\beta$-barrel protein, the GNNQQNY peptide dimer, and models of condensed matter that exhibit structural glass formation and jamming. We have considered measures based on several different properties, namely, the Shannon entropy, an equilibrium thermodynamic measure that uses a sample of local minima, and indices that require additional information about the connections between local minima in the form of transition states. A frustration index is defined that correlates directly with key properties that distinguish relaxation behaviour within this diverse set. The index uses the ratio of the energy barrier to the energy difference with reference to the global minimum. The contributions for each local minimum are weighted by the equilibrium occupation probabilities. Hence we obtain fundamental insight into the connections and distinctions between systems that cover the continuum from efficient structure-seekers to landscapes that exhibit broken ergodicity and rare event dynamics. Published by AIP Publishing. [http://dx.doi.org/10.1063/1.4977794]
\end{abstract}

\section{INTRODUCTION}

The potential energy landscape approach can provide both novel insight and computational methodology in molecular and condensed matter science. ${ }^{1}$ Some of the most powerful tools in the theory of energy landscapes and the associated numerical methodology are based upon descriptions of structure, dynamics, and thermodynamics formulated in terms of local minima on the landscape, and the transition states and pathways that connect them. ${ }^{1}$ Disconnectivity graphs ${ }^{2,3}$ (see Section II A) can provide an insightful representation of manydimensional landscapes and are constructed using information about minima and transition states, preserving the potential (or free) energy barriers between minima. In particular, such graphs have enabled us to identify characteristic patterns of organisation in the underlying potential energy surface, ${ }^{3}$ which result in efficient self-organisation at one end of the spectrum and glassy behaviour at the other extreme. ${ }^{4,5}$ These are systems with different levels of frustration. Self-organising systems have low levels of frustration and undergo efficient relaxation to the lowest-energy minimum, whereas systems that are highly frustrated may never find this minimum-energy structure.

There is a qualitative difference in the landscapes for systems with different degrees of frustration. All the landscapes visualised for good structure-seekers, including "magic number" clusters such as buckminsterfullerene, $\mathrm{C}_{60}$, naturally occurring proteins, bulk representations of crystalline materials, and self-assembling mesoscopic shell structures,

a)dw34@cam.ac.uk have a unique low-lying free energy minimum, with no competing morphologies separated by high barriers. ${ }^{3}$ The appearance of the corresponding disconnectivity graphs has been likened to a multilevel palm tree, where local minima are separated from the global minimum by small barriers. ${ }^{3}$ The resulting "funnelled" landscape effectively guides relaxation to the global minimum. An example of a palm tree disconnectivity graph is shown for a Lennard-Jones cluster of 13 atoms $\left(\mathrm{LJ}_{13}\right)$ in the first panel of Figure 1. At the other extreme, the landscape for structural glass formers supports an exponentially large number of amorphous minima, separated by high barriers. ${ }^{5}$ An example of a glassy tree can be seen in Fig. 3.

The folding of naturally occurring proteins has been associated with a "principle of minimal frustration." teins fold easily and as they do so, their energy decreases more than would be expected by chance. There is a strong energetic bias toward the native state. This bias overcomes the unfavourable entropy change and also any kinetic trap contained within the structure of the landscape. Geometrical frustration, for example associated with the fact that locally favoured structures, such as icosahedra, cannot tile space, is considered to play a key role in the glass transition. ${ }^{7}$ Between the limits of efficient self-organisation and glassy behaviour lies a continuous range of complexity. Of particular interest are systems characterised by two competing low-energy structures with a large interconversion barrier. Such "double funnel" landscapes may exhibit a separation of relaxation time scales and heat capacity features associated with a low temperature transition between the alternative morphologies. ${ }^{3}$ This competition between structures has been termed "frustration," 8,9 and a number of examples have been analysed in detail, as benchmarks for global optimisation, enhanced 


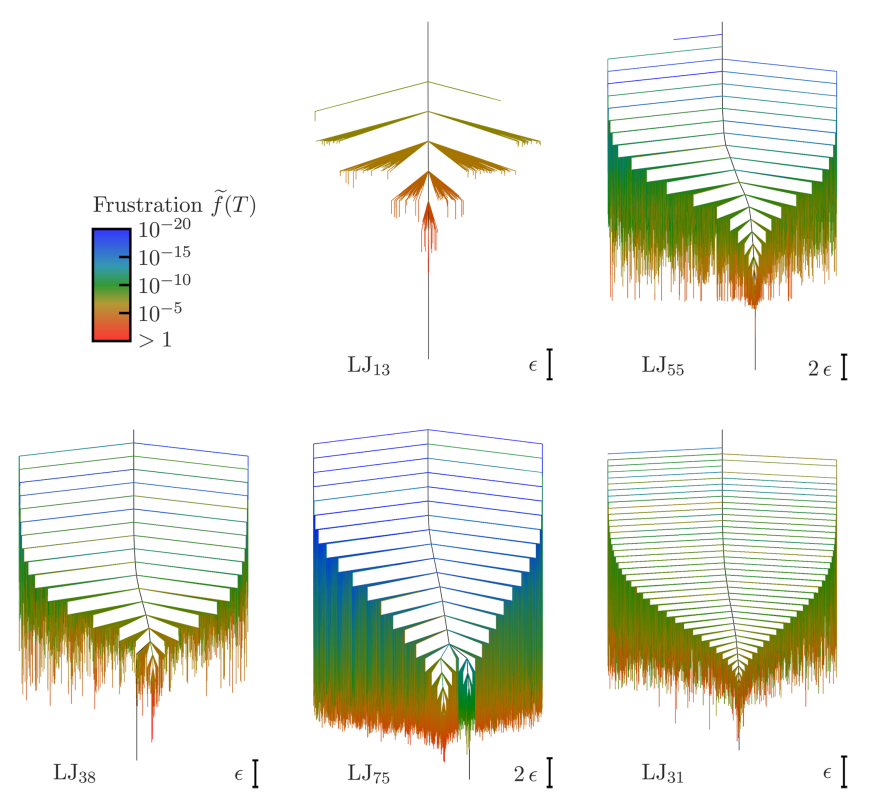

FIG. 1. Disconnectivity graphs for five Lennard-Jones clusters. The graphs are arranged in the order of increasing frustration from left to right and top to bottom: $\mathrm{LJ}_{13}, \mathrm{LJ}_{55}, \mathrm{LJ}_{38}, \mathrm{LJ}_{75}$, and $\mathrm{LJ}_{31}$. The global minimum is shown in black, and branches leading to every other minimum are coloured according to their contribution to the $\widetilde{f}(T)$ frustration index at the melting temperature of the system. The scale bar for each graph defines the energy spacing.

thermodynamic sampling designed to overcome broken ergodicity, and rare event dynamics. ${ }^{3,10-15}$

A variety of disconnectivity graphs for contrasting energy landscapes will be presented in Sec. III. Our aim is to show how the global organisation of the landscape can be described in a quantitative manner, by considering various indices that reflect different measures of the complexity or frustration. One particular frustration index is found to be particularly suitable for this purpose.

\section{THE POTENTIAL ENERGY LANDSCAPE}

The potential energy landscape (PEL) is a function of all the relevant atomic or molecular coordinates, which we represent by the components of a vector $\mathbf{X}$. Here we coarse-grain by considering stationary points, where the energy gradient vanishes, using second derivatives to classify minima, transition states, and higher-index saddles. The PEL is then characterised by three quantities: the potential energy $V(\mathbf{X}) ; \mathbf{g}(\mathbf{X})$, the first derivative of the potential energy; and the second derivative matrix $\mathbf{H}(\mathbf{X})$. The elements of the Hessian $\mathbf{H}(\mathbf{X})$ define the curvature of the landscape.

Stationary points have a physical meaning. Local minima have no negative Hessian eigenvalues, and a small displacement in any of the internal coordinates increases the energy. Even apparently simple systems can support large numbers of local minima on the PEL; the lowest is the global minimum. Transition states are defined geometrically in the present work, as stationary points with a single negative Hessian eigenvalue. Most transition states connect two minima, which are identified by calculating (approximate) steepest-descent paths leaving parallel and antiparallel to the eigenvector corresponding to the negative eigenvalue; such a minimum-transition state-minimum triplet can be considered as an elementary rearrangement.

Knowledge of all the stationary points in the system would in principle provide insight into a variety of interesting properties. However, larger systems possess a huge number of stationary points, and so some method of sampling the landscape to obtain a representative selection is required. There are a number of possible approaches to this problem and the most appropriate method will generally depend on the properties of interest. Although the landscape was explored in different ways for the different systems considered in this paper, in all cases the same geometry optimisation tools were employed for locating minima and transition states and for characterising pathways. The OPTIM program was used for all these calculations.

Local minima were found by minimising the potential energy using the limited memory Broyden-Fletcher-GoldfarbShanno (LBFGS) algorithm, ${ }^{16}$ which has proved to be particularly efficient. ${ }^{17}$ Transition states were located using the doubly-nudged $^{18}$ elastic band ${ }^{19,20}$ (DNEB) method. Local maxima in the chain of states corresponding to the DNEB interpolation were taken as candidate transition states (TSs), which were refined using hybrid eigenvector-following. ${ }^{21}$ To identify the two minima connected by each TS, a small step was taken both parallel and antiparallel to the eigenvector corresponding to the unique negative Hessian eigenvalue. LBFGS minimisation was then applied to each set of displaced coordinates to find the two connected minima. If a complete pathway of elementary arrangements between the two original minima was not found, a modified Dijkstra algorithm ${ }^{22}$ was used to choose a pair of intermediate minima to connect next, with the aim of completing the pathway using the smallest number of connection attempts.

For most of the systems studied in the present work, the PATHSAMPLE program was used as a driver for OPTIM to expand the stationary point databases. The discrete path sampling (DPS) approach ${ }^{13}$ implemented in PATHSAMPLE is a coarse-grained analog of the transition path sampling method. ${ }^{23}$ PATHSAMPLE can systematically generate sets of discrete paths from an initial connected path between two end points, creating a kinetic transition network. These discrete paths are connected sequences of minima and intervening transition states. ${ }^{1,13}$ The PELE $^{24}$ package, a python library for finding global minima and connecting minima, was also used for some of the systems. Further details are provided below.

\section{A. Disconnectivity graphs}

To construct a disconnectivity graph, we group minima that are mutually accessible at a given total energy, $E$. These minima are connected by single- or multi-step pathways where the energy never exceeds $E$. Minima are placed in a different group if the path between them contains a transition state that lies above the threshold energy, $E$. These groups can then be connected at a higher energy. Each group (or superbasin) is represented by a point, or node, on the horizontal axis, and the vertical axis corresponds to increasing energy. Nodes are joined at higher energy if they belong to the same superbasin and nodes continue down to the level of each single local minimum. The 
horizontal axis is usually arbitrary and the order and spacings between nodes can be chosen for clarity. The superbasin analysis is performed at a discrete series of total energies, $E$. There remains an adjustable parameter in the spacing between energy levels. If the spacing is too large, little topographical information is left, but if it is too small, any coarse-grained structure may be hidden. We emphasise that disconnectivity graphs are simply employed as a visualisation tool to provide insight into the organisation of the potential or free energy landscape. When thermodynamic or kinetic properties are required, we utilise additional information. Connected databases of minima and transition states define kinetic transition networks, which can be constructed systematically using the discrete path sampling (DPS) framework. ${ }^{13}$ Information from the underlying kinetic transition network, specifically the local densities of states, allows us to estimate occupation probabilities, free energies, and minimum-to-minimum rate constants.

\section{SYSTEMS}

\section{A. Atomic clusters}

Many small atomic clusters exhibit efficient relaxation to the global minimum. However, clusters of particular sizes can exhibit two (or more) competing morphologies, distinct structures with similar energy to the global minimum, leading to a frustrated landscape. ${ }^{8,9}$ The interatomic potential considered is the pairwise additive, isotropic Lennard-Jones (LJ) form

$$
V=4 \epsilon \sum_{i<j}\left[\left(\frac{\sigma}{r_{i j}}\right)^{12}-\left(\frac{\sigma}{r_{i j}}\right)^{6}\right],
$$

where $\epsilon$ and $2^{1 / 6} \sigma$ are the pair equilibrium well depth and separation, respectively, and we employ reduced units of energy and distance defined by $\epsilon$ and $\sigma$.

Disconnectivity graphs for five different cluster sizes are shown in Figure 1. For clusters with 13 and 55 atoms, denoted $\mathrm{LJ}_{13}$ and $\mathrm{LJ}_{55}$, the global minimum is a complete Mackay icosahedron, and the landscapes have a single funnel "palm tree" form, with no competing alternative low energy morphologies. ${ }^{3}$ In contrast, for each of $\mathrm{LJ}_{31}, \mathrm{LJ}_{38}$, and $\mathrm{LJ}_{75}$ there is a double funnel structure. For $\mathrm{LJ}_{31}$, the competing funnel is characterised by an alternative surface structure. For $\mathrm{LJ}_{38}$ and $\mathrm{LJ}_{75}$, structures in the competing funnels have fundamentally different packing schemes, separated from higher energy incomplete icosahedral minima by relatively large barriers. ${ }^{3,10}$ The aim of this paper is to find a useful measure of this frustration, which should identify the $\mathrm{LJ}_{31}, \mathrm{LJ}_{38}$, and $\mathrm{LJ}_{75}$ double-funnel systems as significantly more frustrated than $\mathrm{LJ}_{13}$ and $\mathrm{LJ}_{55}$. When searching for the global minimum, the mean first encounter time is significantly longer for systems with a double funnel structure. ${ }^{1,25}$ Hence, these double funnel landscapes provide important benchmarks for global optimisation, enhanced sampling schemes, and rare event methods. ${ }^{1-15}$

Stationary point databases were all obtained by initially connecting low-lying minima encountered in basin-hopping global optimisation runs. ${ }^{26,27}$ Further refinement was based on locating kinetically relevant discrete paths between competing morphologies for the double-funnel systems or increasing the number of connections per minimum for $\mathrm{LJ}_{13}$ and $\mathrm{LJ}_{55}$. In each case, the database was also enlarged using a scheme to remove artificial frustration. ${ }^{28}$ Aside from $\mathrm{LJ}_{13}$, the samples obtained are necessarily incomplete. These databases were therefore extended until the appearance of the lowenergy region of the landscape appeared to have converged, as judged by inspection of the disconnectivity graphs. The convergence of the various measures of frustration defined in Section IV for the lower temperature range of interest was also monitored.

\section{B. $\left(\mathrm{H}_{2} \mathrm{O}\right)_{20}$}

The energy landscape of the "nanodroplet" cluster $\left(\mathrm{H}_{2} \mathrm{O}\right)_{20}$ displays signatures of hierarchical organisation. ${ }^{3}$ This complexity has been associated with the additional rotational degrees of freedom for the water molecule, which lead to a wide range of energies and barrier heights separating structures with similar arrangements of the oxygen atoms, but alternative hydrogen-bonding patterns. ${ }^{3}$ Understanding how these effects result in qualitative differences from the atomic clusters considered above is of great interest in view of the central role of water in the solvation of biomolecular systems.

For the TIP4P rigid molecule pair potential, ${ }^{29}$ the global potential energy minimum can be described in terms of three pentagonal prisms sharing approximately square faces. ${ }^{30,31}$ There are two other morphologies with competing low energy minima based on face-sharing cuboids ("box-kite" structure $^{32}$ ) and three stacked pentagonal prisms sharing pentagonal faces. ${ }^{31}$ Minima based on dodecahedral structures generally lie rather higher in energy. ${ }^{31}$ The landscape for this system was explored as follows. Four low-energy structures corresponding to the different morphologies were connected and then the pathway between the two lowest structures was further refined using a short-cutting procedure. ${ }^{28,33}$ The resulting disconnectivity graph is shown in Fig. 2.

\section{A model $\beta$-barrel protein}

Here we considered a coarse-grained BLN model for a 69-residue protein, ${ }^{34}$ where each amino acid is represented by a hydrophobic (B), hydrophilic (L), or neutral (N) bead. Stiff harmonic springs were employed to restrain the bond lengths. ${ }^{35}$ The global minimum of the sequence $\mathrm{B}_{9} \mathrm{~N}_{3}(\mathrm{LB})_{4} \mathrm{~N}_{3} \mathrm{~B}_{9} \mathrm{~N}_{3}(\mathrm{LB})_{4} \mathrm{~N}_{3} \mathrm{~B}_{9} \mathrm{~N}_{3}(\mathrm{LB})_{5} \mathrm{~L}\left(\mathrm{BLN}_{69}\right)$ is a sixstranded $\beta$-barrel, with three chains forming a hydrophobic core. However, we have previously shown that there are a number of low-lying minima with alternative arrangements of the $\beta$-strands, separated by high barriers. ${ }^{36}$

Figure 2 shows a disconnectivity graph for $\mathrm{BLN}_{69}$. The database was again produced by initially connecting lowlying minima obtained using basin-hopping global optimisation, ${ }^{26,27}$ and then refining to locate kinetically relevant paths between alternative morphologies and remove artificial frustration caused by undersampling.

\section{The amyloidogenic GNNQQNY peptide dimer}

The polar amyloidogenic heptapeptide GNNQQNY is a key component of the $\mathrm{N}$-terminal prion-determining domain of the yeast protein Sup35. This peptide is noteworthy for its 


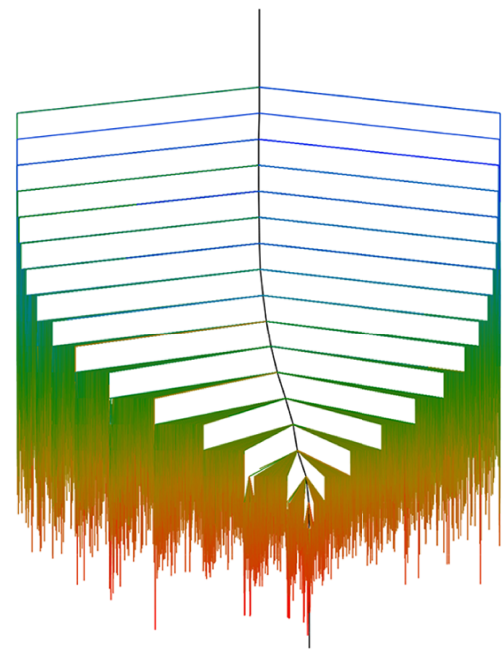

$\left(\mathrm{H}_{2} \mathrm{O}\right)_{20}$

$20 \epsilon \Gamma$
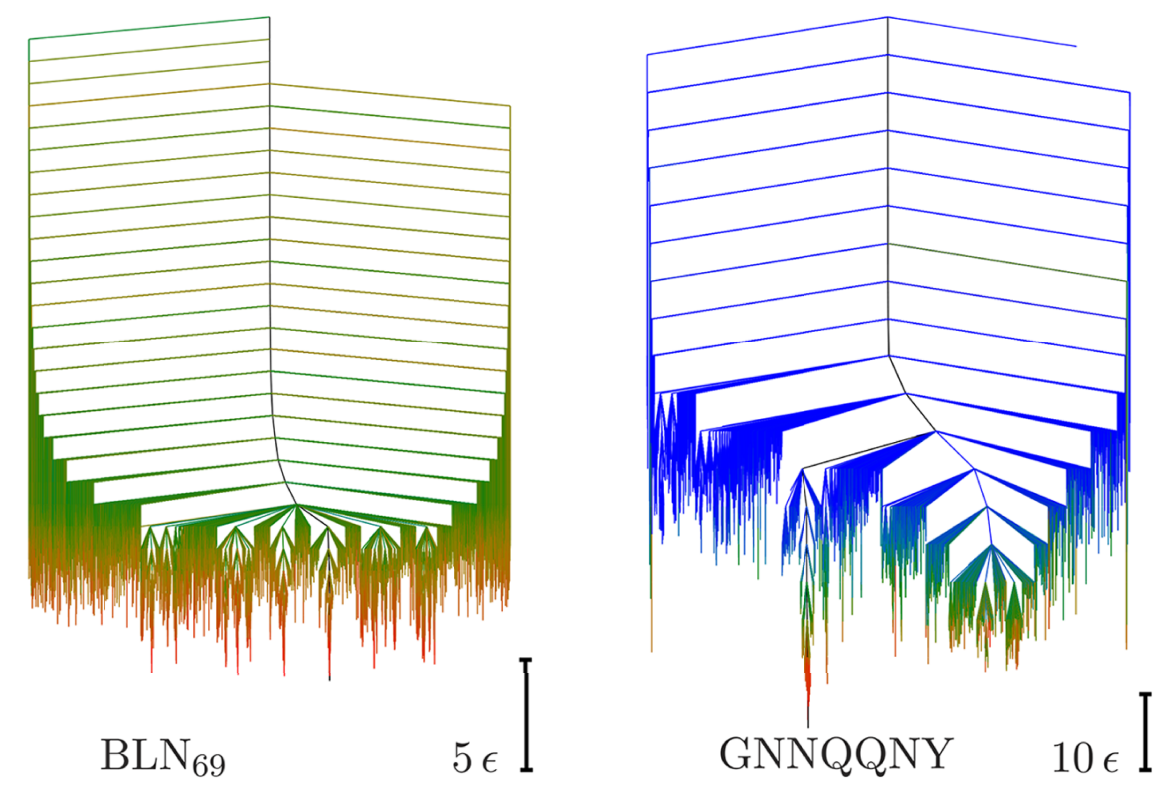

FIG. 2. Disconnectivity graphs for $\left(\mathrm{H}_{2} \mathrm{O}\right)_{20}$, the 69-residue BLN model protein, and the heptapeptide GNNQQNY. The global minimum for each system is shown in black and branches leading to every other minimum are coloured according to their contribution to the $\widetilde{f}(T)$ frustration index at the melting temperature of the system. The colour scale is identical to that used in Figure 1, where minima corresponding to red branches make the largest contribution to frustration. The scalebar for each graph defines the energy spacing.
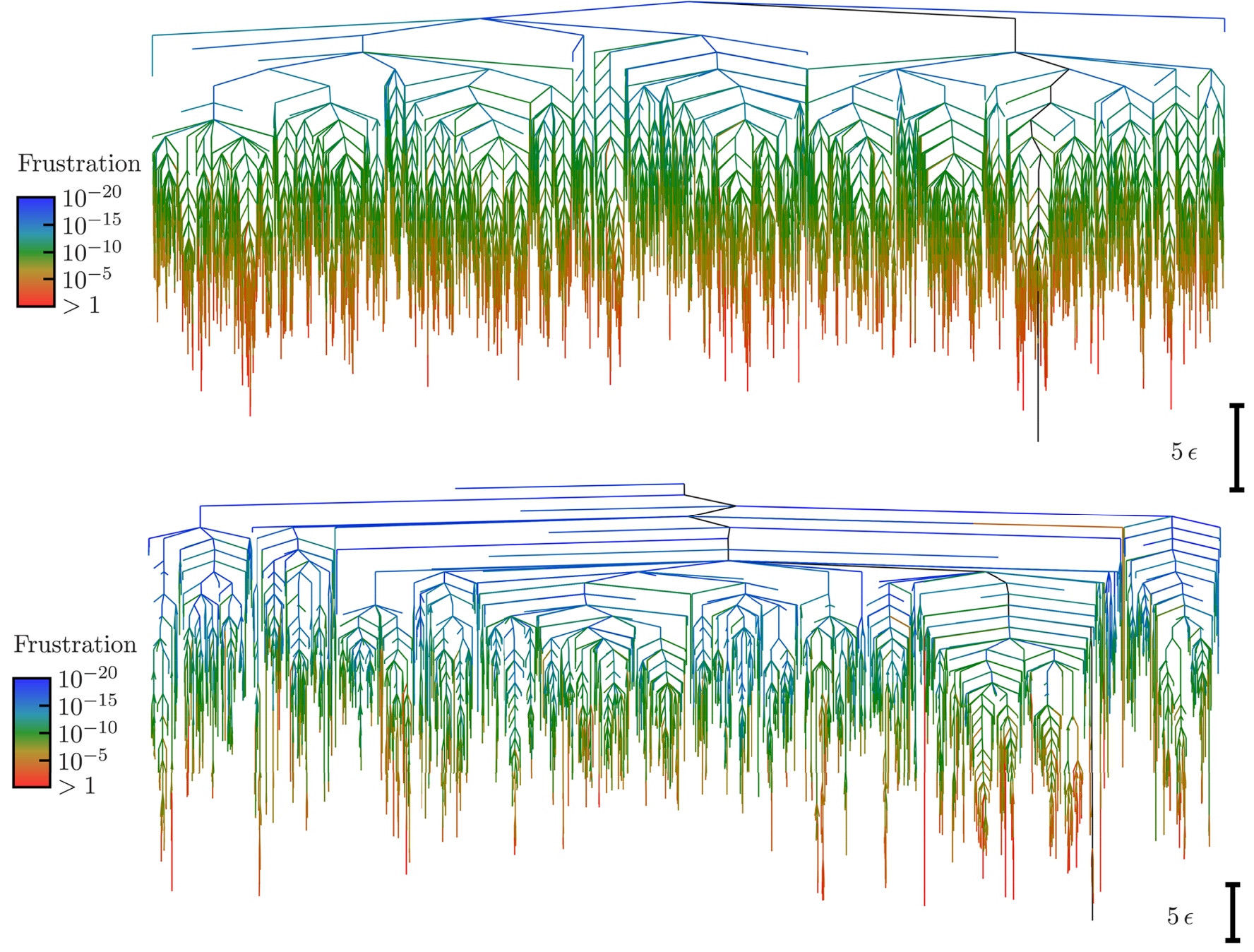

FIG. 3. Disconnectivity graphs for $\mathrm{BLJ}_{60}$ (top) and $\mathrm{BLJ}_{256}$ (bottom). The colour scale shows the contributions to the frustration index $\widetilde{f}(T)$ at the melting temperature, and the energy scale bar defines the spacing. 
individual stability, ${ }^{37}$ and a crystal structure has been characterised, where the monomers align to give in-register parallel $\beta$-sheets. ${ }^{38}$ Here we employ a DPS database created for the GNNQQNY dimer, which we previously found to support four competing conformations: one that is intermediate between compact and extended structures and three $\beta$-sheets corresponding to in-register parallel, off-register parallel, and antiparallel arrangements. ${ }^{28}$ These results were obtained with the CHARMM $19^{39}$ potential and EEF1 implicit solvent. ${ }^{40}$ The corresponding potential energy landscape might be expected to exhibit an intermediate level of frustration, and this intuition is consistent with the new results reported below. The time scale for interconversion between the four low-lying structures was estimated as hours or longer at $298 \mathrm{~K} .{ }^{28}$ Replica exchange molecular dynamics ${ }^{41}$ (REMD) was used for an initial exploration of the conformational space. The resulting database of minima and transition states was then grown using DPS focusing on the paths making the largest contributions to the steady state rate constants between the alternative morphologies. Shortcutting procedures ${ }^{28,33}$ were followed by the removal of artificial traps. ${ }^{28}$ A disconnectivity graph for this system is shown in Fig. 2.

\section{E. Binary structural glass formers}

Binary Lennard-Jones (BLJ) potentials are extensively used in studies of structural glass phenomenology, ${ }^{42-45}$ since with suitable parameterisations they do not crystallise on time scales usually accessible to molecular dynamics. Here we consider BLJ systems modelled using periodic boundary conditions containing two types of atom, $\mathrm{A}$ and $\mathrm{B}$, in the ratio $80: 20$, which was originally proposed to model the metallic glass $\mathrm{Ni}_{0.8} \mathrm{P}_{0.2}{ }^{46}$ Although this model possesses mixed and phase separated crystalline structures, ${ }^{47,48}$ these can easily be excluded from the structural databases employed for landscape analysis. The parameterisation employed was $\sigma_{\mathrm{AA}}=1$, $\sigma_{\mathrm{AB}}=0.8, \sigma_{\mathrm{BB}}=0.88, \epsilon_{\mathrm{AA}}=1, \epsilon_{\mathrm{AB}}=1.5$, and $\epsilon_{\mathrm{BB}}=0.5 .{ }^{42}$ The resulting disconnectivity graphs ${ }^{5}$ exhibit many low-lying minima corresponding to amorphous structures, separated by high barriers of order $30 k_{B} T_{g}$, where $T_{g}$ corresponds to a glass transition temperature. Grouping local minima according to whether they can be interconverted without a cage-breaking rearrangement, which would be necessary for diffusion, has revealed a higher order structure in the energy landscape, ${ }^{5}$ which may yield a useful way to define "metabasins." 49,50
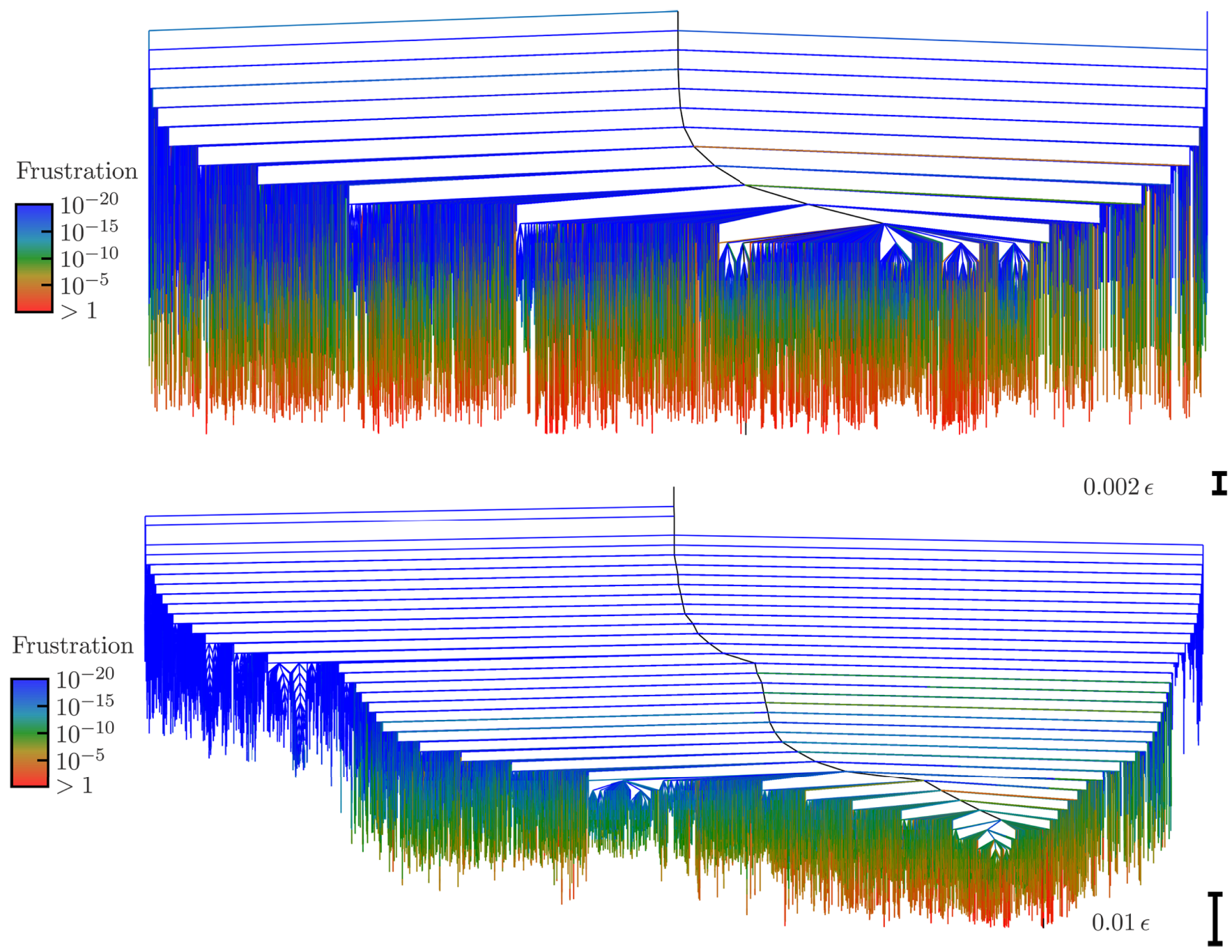

FIG. 4. Disconnectivity graphs for 60 (top) and 256 (bottom) soft spheres. The colour scale shows the contributions to the frustration index $\widetilde{f}(T)$ at the melting temperature, and the scalebar defines the energy spacing. 
To avoid finite-size effects, the system must not be too small, and 60 atoms were found to be a good compromise in previous work. ${ }^{43}$ Our results are for a 60 -atom BLJ mixture of 48 type $\mathrm{A}$ and 12 type $\mathrm{B}$ particles $\left(\mathrm{BLJ}_{60}\right)$ and a 256-atom mixture of $204 \mathrm{~A}$ and $52 \mathrm{~B}$ particles $\left(\mathrm{BLJ}_{256}\right)$. Both systems have a number density of 1.3 in reduced units of $\sigma_{\mathrm{AA}}^{-3}$ (used throughout) and were simulated with periodic boundary conditions. Full details are given in Ref. 51 and disconnectivity graphs for $\mathrm{BLJ}_{60}$ and $\mathrm{BLJ}_{256}$ are shown in Figure 3. The $\mathrm{BLJ}_{60}$ database contains over 11000 minima, while the database for $\mathrm{BLJ}_{256}$ has 2500 . The PELs for both systems show typical glassy organisation, with many minima of similar energies separated by high barriers, and a hierarchical structure. For both BLJ systems, initial samples of minima were obtained from locally ergodic canonical MD trajectories. ${ }^{51}$ Local minimisation was applied for each configuration of the trajectory, producing a sequential series of minima. Adjacent minima were then connected, giving a single kinetically relevant discrete path. ${ }^{5,52}$ We expect to find lower frustration for such a connected path of minima and transition states, compared to a more extensive search for a particular region of the energy landscape, as discussed below.

\section{F. Soft spheres: Landscapes for jamming}

Jamming corresponds to a transition to rigidity in disordered matter. In the jammed state, materials respond essentially elastically to small applied shear stresses. However, such systems can easily be unjammed and made to flow by tuning various control parameters. The jamming transition can be induced by varying thermodynamic variables, such as temperature or density, and also by mechanical variables, such as applied stress. ${ }^{53}$

Packings of soft repulsive spheres at zero temperature exhibit a sharp jamming transition in the thermodynamic limit. The transition is induced by applied pressure or enforced packing fraction, causing deformation of the particles. When deformations vanish, the system loses rigidity. At a low packing fraction, no particles interact, a state described as an unjammed "mechanical vacuum." As the packing fraction is increased, there is a transition to a jammed, rigid structure. Such frictionless spheres with finite-range repulsions have been studied extensively. ${ }^{53-55}$

The two systems considered here consist of $N=60$ and $N=256$ spheres of equal mass, $M$, and variable radii, $R_{i}$, with periodic boundary conditions. The radii are distributed evenly within the range 0.416 to 0.583 . When they overlap, the spheres interact via a Hertzian soft sphere potential. Denoting the centre-to-centre distance as $r_{i j}$, a dimensionless overlap parameter, $\delta_{i j}$, can be defined as

$$
\delta_{i j}=1-\frac{r_{i j}}{R_{i}+R_{j}} .
$$

The interaction potential is zero unless $\delta_{i j}>0$ and then has the form

$$
V_{i j}=\epsilon_{i j} \delta_{i j}^{2.5} .
$$

For the soft sphere systems, a particular region of the potential energy landscape was explored in detail using PELE. Basin-hopping steps were taken from an initial starting minimum to find further minima, and then pathways were obtained using DNEB. The search then returns to the initial starting minimum for further basin-hopping steps. The resulting database was refined with the untrapping method designed to remove artificial frustration, as for some of the other models discussed above. ${ }^{28}$ The untrapping scheme was designed to locate missing lower energy pathways in the low energy part of the landscape. However, for soft spheres, it also results in significant further exploration, finding many more minima with similar energies, which contribute to the frustration. As this sampling method may overestimate frustration, for comparison we have performed an identical analysis for $\mathrm{BLJ}_{256}$. Disconnectivity graphs are shown in Fig. 4 for the 60 and 256 sphere systems and in Fig. 5 for $\mathrm{BLJ}_{256}$.

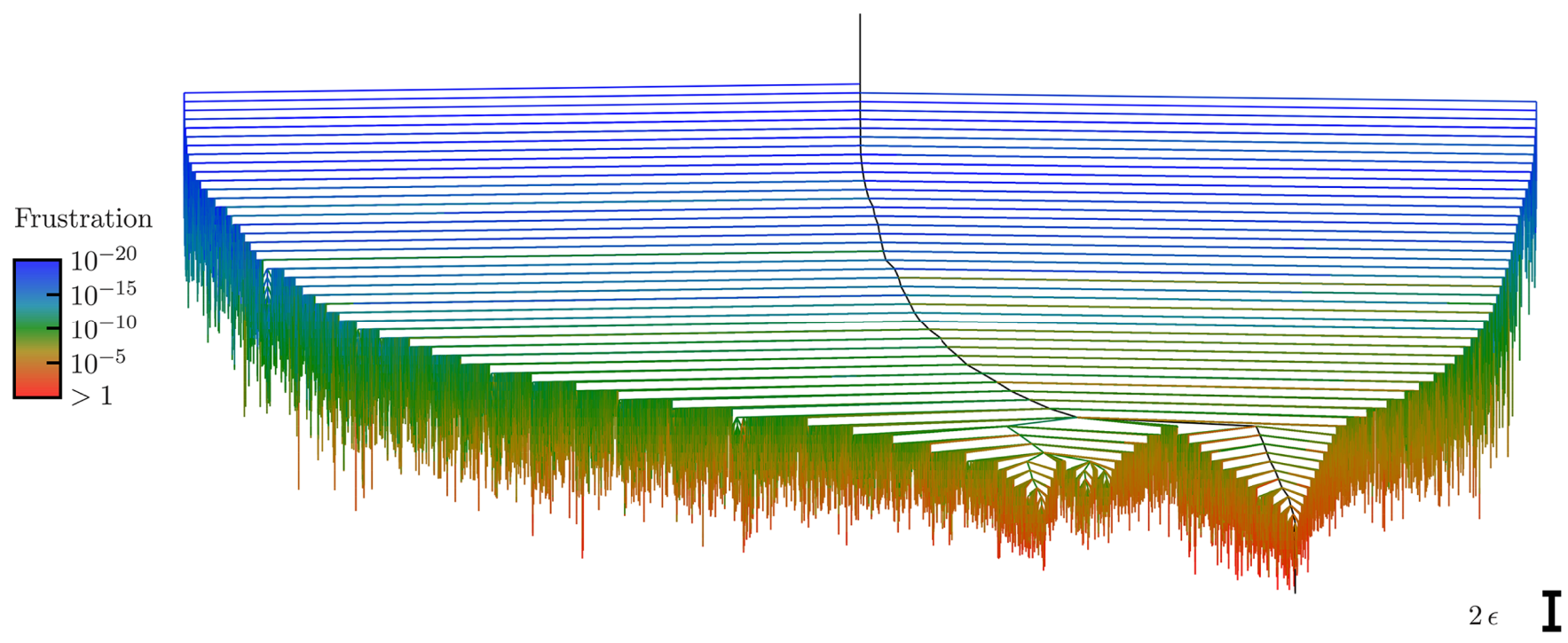

FIG. 5. Disconnectivity graph corresponding to extensive exploration of a local region for $\mathrm{BLJ}_{256}$. The colour scale shows the contributions to the frustration index $\widetilde{f}(T)$ at the melting temperature, and the scalebar defines the energy spacing. 


\section{QUANTIFYING FRUSTRATION IN THE LANDSCAPE}

We have considered various different indices to analyse the degree of frustration in different systems. First, the Shannon entropy, ${ }^{56,57} s(T)$, depends upon equilibrium thermodynamic properties, and therefore only requires information about local minima

$$
s(T)=-\sum_{\alpha} p_{\alpha}^{\mathrm{eq}}(T) \ln p_{\alpha}^{\mathrm{eq}}(T),
$$

where $T$ is the temperature and $p_{\alpha}^{\mathrm{eq}}(T)$ is the equilibrium occupation probability of minimum $\alpha$, calculated using harmonic vibrational densities of states. ${ }^{58}$ If information about transition states is not available, this index can provide a useful measure of frustration, as shown in Figure 6. To compare results for very different systems, we have used a dimensionless temperature, obtained using an estimate for the melting temperature, $T_{m}$. This estimate comes from the temperature at the corresponding heat capacity maximum, obtained consistently within the harmonic superposition approximation. ${ }^{58}$

The three indices defined below require databases where the connectivity of the local minima is defined by transition states

$$
\begin{aligned}
N(T) & =\sum_{\alpha=1}^{m} \sum_{\gamma<\alpha}\left[p_{\alpha}^{\mathrm{eq}}(T)+p_{\gamma}^{\mathrm{eq}}(T)\right] n_{\alpha \gamma}(T), \\
K(T) & =\sum_{\alpha=1}^{m} \sum_{\gamma<\alpha}\left[p_{\alpha}^{\mathrm{eq}}(T) k_{\gamma \alpha}(T)+p_{\gamma}^{\mathrm{eq}}(T) k_{\alpha \gamma}(T)\right], \\
f(T) & =\sum_{\alpha \neq \mathrm{gmin}} p_{\alpha}^{\mathrm{eq}}(T)\left(\frac{V_{\alpha}^{\dagger}-V_{\mathrm{gmin}}}{V_{\alpha}-V_{\mathrm{gmin}}}\right) .
\end{aligned}
$$

$N(T)$ uses $n_{\alpha \gamma}$, the number of steps (transition states) in the fastest path between minima $\gamma$ and $\alpha$, and $K(T)$ uses $k_{\alpha \gamma}$, the rate constant for transitions between minima $\gamma$ and $\alpha . n_{\alpha \gamma}$ and $k_{\alpha \gamma}$ were both obtained from the Dijkstra shortest path analysis, ${ }^{59}$ with edge weights of $-\ln P_{\alpha \gamma}$, where $P_{\alpha \gamma}$ is the branching probability of stepping to minimum $\alpha$ from among the direct connections of minimum $\gamma$. The branching probabilities and minimum-to-minimum rate constants $k_{\alpha \gamma}$ were estimated from transition state theory, ${ }^{60-62}$ using harmonic vibrational densities of states. In contrast, $f(T)$ uses information about the potential energy of the minima, $V_{\alpha}$, and

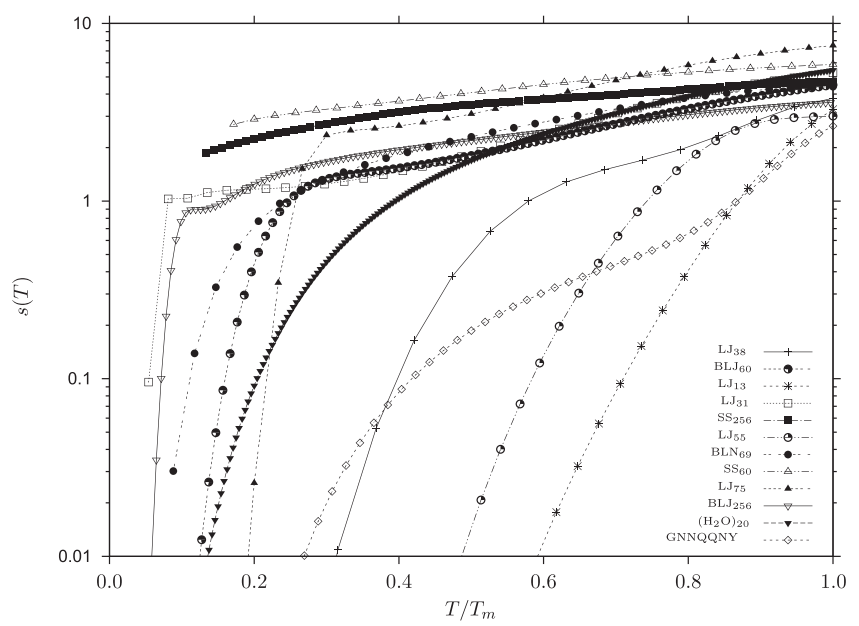

FIG. 6. The Shannon entropy, $s(T)$, for all the models considered. transition states. $V_{\text {gmin }}$ signifies the potential energy of the lowest minimum in the database and $V_{\alpha}^{\dagger}$ is the potential energy of the highest transition state on the lowest energy path between minimum $\alpha$ and the lowest minimum. The lowest minimum is the global minimum for most of the databases considered, except for the condensed matter systems, where the crystalline region of configuration space is intentionally excluded.

The most insightful quantity was found to be $f(T)$, which employs barrier thresholds $V_{\alpha}^{\dagger}$ in the same way as a scheme that we introduced in earlier work to help refine databases to remove artificial frustration. ${ }^{28}$ This approach was, in turn, derived from an analogous index based on free energies that we used earlier, ${ }^{63}$ which extends measures based on stability ${ }^{64}$ and energy gaps ${ }^{65,66}$ by including explicit barrier information. A further advantage of $f(T)$ over the other measures that include connectivity, $N(T)$ and $K(T)$, is that it does not require additional characterisation of discrete paths or rate information and hence, it is quick and easy to calculate.

We calculated $V_{\alpha}^{\dagger}$ in tandem with the superbasin analysis ${ }^{2}$ that yields the disconnectivity graphs, identifying the energy threshold below which the lowest minimum is no longer accessible from minimum $\alpha$. Results for $f(T)$, which we will refer to as the frustration index, are shown in Figure 7.

Analogous indices $\widetilde{s}(T), \widetilde{N}(T), \widetilde{K}(T)$, and $\widetilde{f}(T)$ were calculated from the probabilities $\widetilde{p}_{\alpha}^{\mathrm{eq}}=p_{\alpha}^{\mathrm{eq}} /\left(1-p_{\mathrm{gmin}}^{\mathrm{eq}}\right)$. These quantities reflect the renormalised relative populations of the minima when the temperature dependence of the global minimum is removed. As the temperature decreases, the equilibrium occupation probability of the global minimum can become very large. As the global minimum does not itself contribute to frustration, this high occupation probability causes the frustration measures to decrease rapidly at low temperature. This decrease is an erroneous effect as relaxation to the global minimum becomes more difficult and hence frustration increases at low temperature. The renormalisation is shown for $\mathrm{LJ}_{38}$ in Figure 8, where $f(T)$ and $\widetilde{f}(T)$ are compared. Full results for $\widetilde{f}(T)$ are shown in Figure 9.

The classification of systems is probably clearest for $\tilde{f}(T)$, which provides a relatively stable index over quite a wide range of temperature, thanks to removal of the temperature dependence of the lowest minimum. We see that for most of the range $0<T / T_{m}<1$ structure-seekers have $\widetilde{f}(T)<1$, frustrated

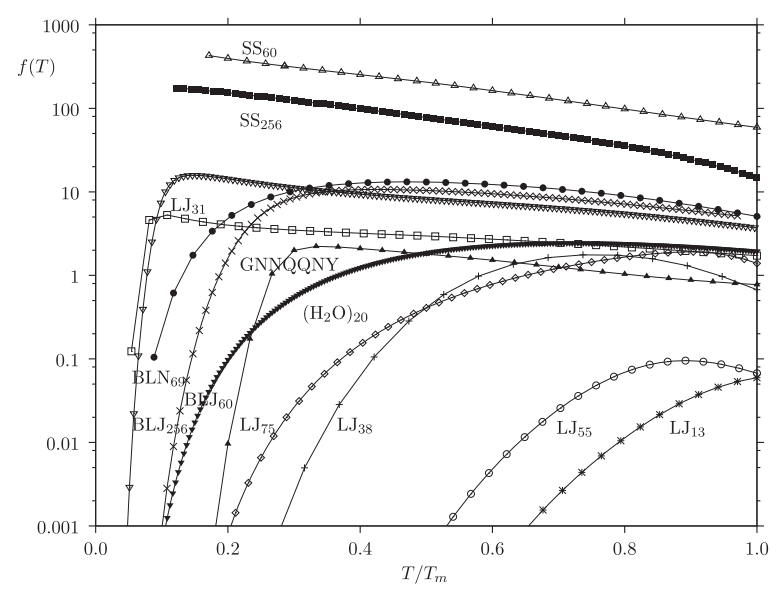

FIG. 7. The frustration index, $f(T)$, is shown for all the models considered. 


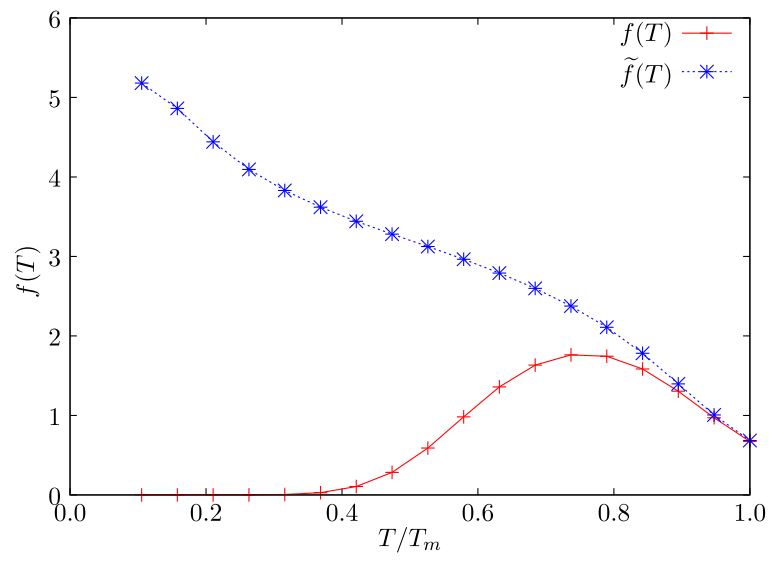

FIG. 8. $f(T)$ and $\widetilde{f}(T)$ for $\mathrm{LJ}_{38}$.

landscapes including structural glasses have $1>\widetilde{f}(T)>50$, and jammed systems have $\widetilde{f}(T)>100$. We would not wish to overinterpret smaller differences in $\widetilde{f}(T)$; it is the order of magnitude differences that are really significant here.

\section{A. Comparison of sampling protocol}

For most of the models compared here, the underlying kinetic transition networks correspond to samples of stationary points designed to describe the dynamics associated with specific pathways or regions of the landscape. The degree of frustration could therefore exhibit some degree of sample dependence, and we have therefore provided details of the landscape exploration methods for each system in Section III. However, if all the landscapes were sampled in the same way, we expect the key trends to be unaffected. A representative sample of minima and transition states should provide us with occupation probabilities and barrier heights that provide a converged value for the chosen measure of frustration, $\widetilde{f}(T)$. The most frustrated systems we have considered here are the soft sphere packings, which were explored through a more extensive local search. The same local exploration was therefore repeated for the binary Lennard-Jones system of 256 particles and continued until we obtained the same number of minima as for the soft sphere example. Fig. 10 shows that the results

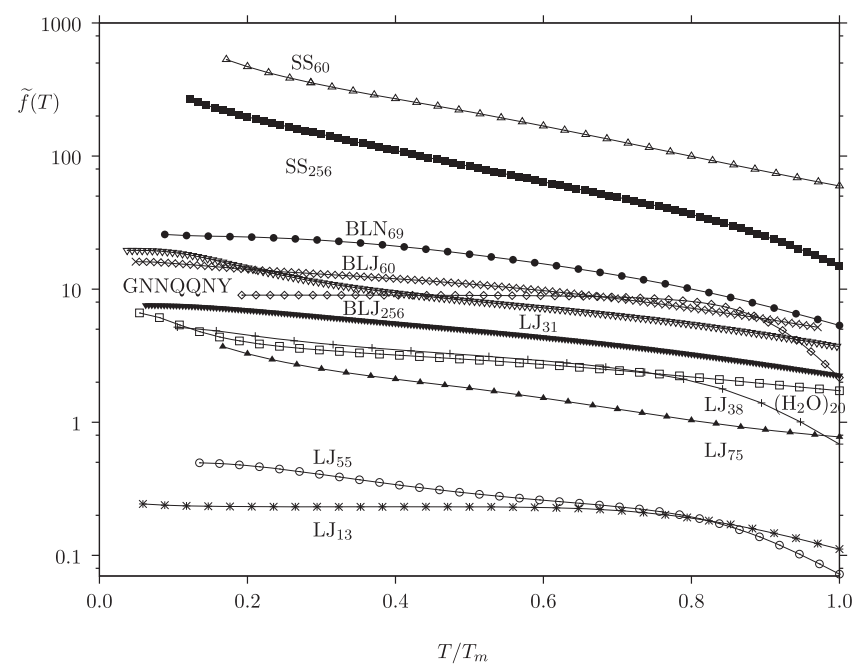

FIG. 9. The frustration index calculated from renormalised probabilities, $\widetilde{f}(T)$, for each of the models considered.

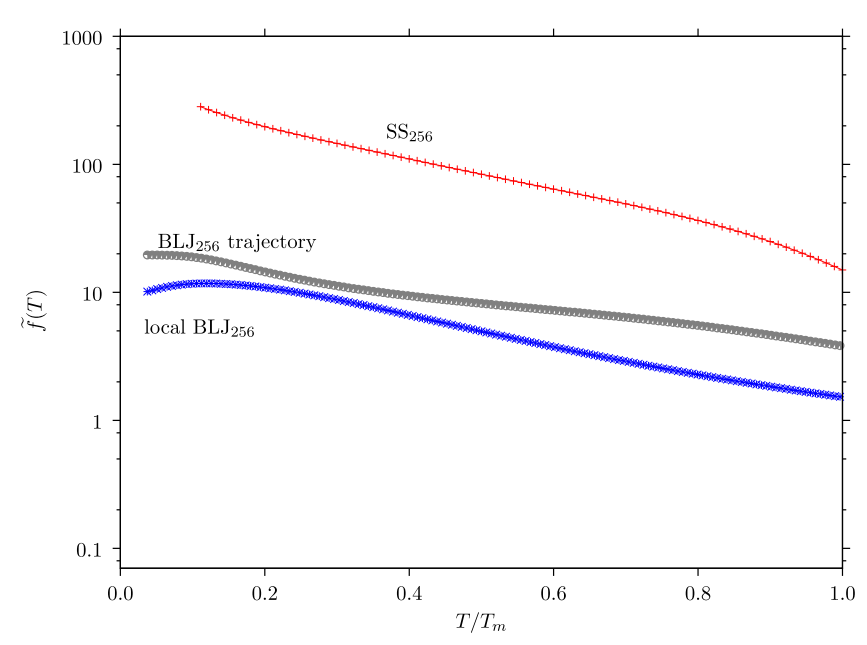

FIG. 10. $\tilde{f}(T)$ for a 256-atom binary Lennard-Jones mixture, where the energy landscape has been explored by two different schemes. In the first approach, the sample is based on a pathway through the landscape corresponding to a locally ergodic molecular dynamics trajectory. In contrast, the second scheme employs a more extensive local exploration, as for the 256-atom soft sphere system.

for this scheme with around 40000 minima are very similar to those obtained for a single kinetically relevant discrete path of around 3600 minima, based upon an initial molecular dynamics trajectory. The frustration index is still significantly lower than that for the soft sphere systems. In fact, the more extensive local searches may produce lower frustration measures as they do not encounter many of the alternative low-energy minima separated by high barriers that are present in the landscape.

\section{DISCUSSION AND CONCLUSIONS}

We have explored measures to quantify the global organisation of the potential energy landscape. A frustration index, $\widetilde{f}(T)$, including renormalised approximate equilibrium occupation probabilities and barrier information, is found to be particularly useful. If transition state information is not available, the Shannon entropy, based on equilibrium occupation probabilities, can also provide a measure of frustration, albeit without allowing for dynamical effects.

We studied a number of different systems and found soft sphere packings to be the most frustrated, followed by binary Lennard-Jones models of supercooled liquids and a 69residue model $\beta$-barrel protein. In all these very frustrated systems, there are a number of low-energy competing structures close in energy to the global minimum. Lower frustration is found for systems with a well-defined global minimum. $\mathrm{LJ}_{31}$ is the most frustrated Lennard-Jones cluster, while the amyloidogenic GNNQQNY heptapeptide and the $\left(\mathrm{H}_{2} \mathrm{O}\right)_{20}$ cluster exhibit comparable values for $\widetilde{f}(T)$. The least frustrated systems we compared are the $\mathrm{LJ}_{13}$ and $\mathrm{LJ}_{55}$ clusters, where the landscapes have a single funnel with no competing low-energy structures.

The frustration index provides a quantity to characterise aspects of the potential energy landscape that have previously been described more qualitatively. It is easy to calculate, and we have shown that it reliably distinguishes different degrees of frustration for a variety of systems. The key features are also relatively robust in terms of how the underlying landscape has 
been explored. These results should provide a useful tool for quantifying frustration across very different systems of interest, potentially providing new insight into our understanding of emergent properties.

\section{ACKNOWLEDGMENTS}

We acknowledge the Engineering and Physical Sciences Research Council, UK (EPSRC) for funding under Programme Grant No. EP/I001352/1 and the European Research Council (ERC). Additional data related to this publication are available at the University of Cambridge data repository (https://doi.org/10.17863/CAM.8348). We thank Carl Goodrich for assistance with the soft sphere systems.

${ }^{1}$ D. J. Wales, Energy Landscapes (Cambridge University Press, Cambridge, 2003).

${ }^{2}$ O. M. Becker and M. Karplus, J. Chem. Phys. 106, 1495 (1997).

${ }^{3}$ D. J. Wales, M. A. Miller, and T. R. Walsh, Nature 394, 758 (1998).

${ }^{4}$ D. J. Wales, Philos. Trans. R. Soc., A 363, 357 (2005).

${ }^{5}$ V. K. de Souza and D. J. Wales, J. Chem. Phys. 129, 164507 (2008).

${ }^{6}$ J. D. Bryngelson and P. G. Wolynes, Proc. Natl. Acad. Sci. U. S. A. 84, 7524 (1987).

${ }^{7}$ G. Tarjus, S. A. Kivelson, Z. Nussinov, and P. Viot, J. Phys.: Condens. Matter 17, R1143 (2005).

${ }^{8}$ J. D. Bryngelson, J. N. Onuchic, N. D. Socci, and P. G. Wolynes, Proteins: Struct., Funct., Genet. 21, 167 (1995).

${ }^{9}$ J. N. Onuchic, Z. Luthey-Schulten, and P. G. Wolynes, Annu. Rev. Phys. Chem. 48, 545 (1997).

${ }^{10}$ J. P. K. Doye, M. A. Miller, and D. J. Wales, J. Chem. Phys. 110, 6896 (1999).

${ }^{11}$ J. P. Neirotti, F. Calvo, D. L. Freeman, and J. D. Doll, J. Chem. Phys. 112, 10340 (2000).

${ }^{12}$ F. Calvo, J. P. Neirotti, D. L. Freeman, and J. D. Doll, J. Chem. Phys. 112, 10350 (2000).

${ }^{13}$ D. J. Wales, Mol. Phys. 100, 3285 (2002).

${ }^{14}$ V. A. Mandelshtam, P. A. Frantsuzov, and F. Calvo, J. Phys. Chem. A 110, 5326 (2006).

${ }^{15}$ F. Calvo, Phys. Rev. E 82, 046703 (2010).

${ }^{16}$ J. Nocedal, Math. Comput. 35, 773 (1980).

${ }^{17}$ S. T. Chill, J. Stevenson, V. Ruehle, C. Shang, P. Xiao, J. D. Farrell, D. J. Wales, and G. Henkelman, J. Chem. Theory Comput. 10, 5476 (2014).

${ }^{18}$ S. A. Trygubenko and D. J. Wales, J. Chem. Phys. 120, 2082 (2004).

${ }^{19} \mathrm{G}$. Henkelman and H. Jónsson, J. Chem. Phys. 113, 9978 (2000).

${ }^{20}$ G. Henkelman, B. P. Uberuaga, and H. Jónsson, J. Chem. Phys. 113, 9901 (2000).

${ }^{21}$ L. J. Munro and D. J. Wales, Phys. Rev. B 59, 3969 (1999).

${ }^{22}$ J. M. Carr, S. A. Trygubenko, and D. J. Wales, J. Chem. Phys. 122, 234903 (2005).

${ }^{23}$ C. Dellago, P. G. Bolhuis, F. S. Csajka, and D. Chandler, J. Chem. Phys. 108, 1964 (1998).

${ }^{24}$ See https://github.com/pele-python/pele for Pele: Python energy landscape explorer.
${ }^{25}$ D. J. Wales and H. A. Scheraga, Science 285, 1368 (1999).

${ }^{26}$ Z. Li and H. A. Scheraga, Proc. Natl. Acad. Sci. U. S. A. 84, 6611 (1987).

${ }^{27}$ D. J. Wales and J. P. K. Doye, J. Phys. Chem. A 101, 5111 (1997).

${ }^{28}$ B. Strodel, C. S. Whittleston, and D. J. Wales, J. Am. Chem. Soc. 129, 16005 (2007).

${ }^{29}$ W. L. Jorgensen, J. Chem. Phys. 77, 4156 (1982).

${ }^{30}$ D. J. Wales and I. Ohmine, J. Chem. Phys. 98, 7257 (1993).

${ }^{31}$ D. J. Wales and M. P. Hodges, Chem. Phys. Lett. 286, 65 (1998).

${ }^{32}$ C. J. Tsai and K. D. Jordan, Chem. Phys. Lett. 213, 181 (1993).

${ }^{33}$ J. M. Carr and D. J. Wales, J. Chem. Phys. 123, 234901 (2005).

${ }^{34}$ J. D. Honeycutt and D. Thirumalai, Proc. Natl. Acad. Sci. U. S. A. 87, 3526 (1990).

${ }^{35}$ R. S. Berry, N. Elmaci, J. P. Rose, and B. Vekhter, Proc. Natl. Acad. Sci. U. S. A. 94, 9520 (1997).

${ }^{36}$ M. T. Oakley, D. J. Wales, and R. L. Johnston, J. Phys. Chem. B 115, 11525 (2011).

${ }^{37}$ M. Balbirnie, R. Grothe, and D. S. Eisenberg, Proc. Natl. Acad. Sci. U. S. A. 98, 2375 (2001).

${ }^{38}$ R. Nelson, M. R. Sawaya, M. Balbirnie, A. O. Madsen, C. Riekel, R. Grothe, and D. Eisenberg, Nature 435, 773 (2005).

${ }^{39}$ B. R. Brooks, R. E. Bruccoleri, B. D. Olafson, D. J. States, S. Swaminathan, and M. Karplus, J. Comput. Chem. 4, 187 (1983).

${ }^{40}$ T. Lazaridis and M. Karplus, Proteins: Struct., Funct., Genet. 35, 133 (1999).

${ }^{41}$ Y. Sugita and Y. Okamoto, Chem. Phys. Lett. 314, 141 (1999).

${ }^{42}$ W. Kob and H. C. Andersen, Phys. Rev. Lett. 73, 1376 (1994).

${ }^{43}$ S. Büchner and A. Heuer, Phys. Rev. Lett. 84, 2168 (2000).

${ }^{44}$ F. Sciortino, W. Kob, and P. Tartaglia, Phys. Rev. Lett. 83, 3214 (1999).

${ }^{45}$ S. Sastry, Nature 409, 164 (2001).

${ }^{46}$ T. A. Weber and F. H. Stillinger, Phys. Rev. B 31, 1954 (1985).

${ }^{47}$ T. F. Middleton, J. Hernández-Rojas, P. N. Mortenson, and D. J. Wales, Phys. Rev. B 64, 184201 (2001).

${ }^{48}$ J. R. Fernández and P. Harrowell, Phys. Rev. E 67, 011403 (2003).

${ }^{49}$ F. H. Stillinger, Science 267, 1935 (1995).

${ }^{50}$ B. Doliwa and A. Heuer, J. Phys.: Condens. Matter 15, S849 (2003).

${ }^{51}$ V. K. de Souza and D. J. Wales, Phys. Rev. B 74, 134202 (2006).

${ }^{52}$ V. K. de Souza and D. J. Wales, J. Chem. Phys. 130, 194508 (2009).

${ }^{53}$ A. Liu and S. R. Nagel, Annu. Rev. Condens. Matter Phys. 1, 347 (2010).

${ }^{54}$ M. van Hecke, J. Phys.: Condens. Matter 22, 033101 (2009).

${ }^{55}$ C. S. O'Hern, L. E. Silbert, A. J. Liu, and S. R. Nagel, Phys. Rev. E 68, 011306 (2003).

${ }^{56}$ C. E. Shannon, Bell Syst. Tech. J. 27, 379-423 (1948).

${ }^{57}$ C. E. Shannon and W. Weaver, The Mathematical Theory of Communication (University of Illinois Press, Urbana, IL, 1949).

${ }^{58}$ D. J. Wales, Mol. Phys. 78, 151 (1993).

${ }^{59}$ E. W. Dijkstra, Numer. Math. 1, 269 (1959).

${ }^{60}$ H. Pelzer and E. Wigner, Z. Phys. Chem. B15, 445 (1932).

${ }^{61} \mathrm{~W}$. Forst, Theory of Unimolecular Reactions (Academic Press, New York, 1973).

${ }^{62}$ K. J. Laidler, Chemical Kinetics (Harper \& Row, New York, 1987).

${ }^{63}$ J. M. Carr and D. J. Wales, J. Phys. Chem. B 112, 8760 (2008).

${ }^{64}$ A. Godzik, A. Kolinski, and J. Skolnick, J. Comput.-Aided Mol. Des. 7, 397 (1993).

${ }^{65}$ D. U. Ferreiro, J. A. Hegler, E. A. Komives, and P. G. Wolynes, Proc. Natl. Acad. Sci. U. S. A. 104, 19819 (2007).

${ }^{66}$ D. U. Ferreiro, J. A. Hegler, E. A. Komives, and P. G. Wolynes, Proc. Natl. Acad. Sci. U. S. A. 108, 3499 (2011). 